\title{
Role of modified Barium Swallow Pre and Post VitalStim therapy In the rehabilitation of the swallowing mechanism
}

Hoda Salah Darwish ${ }^{1 *}$, Hanaa Ahmed Kamel ${ }^{2}$, Mufeed AL Jeady ${ }^{3}$ and Mohamed Yasser Habash ${ }^{4}$

Correspondence: darwish.hoda@yahoo.com

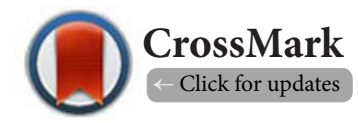

\footnotetext{
${ }^{1}$ Assistant Professor of Radio-Diagnosis, Suez Canal University, Egypt.

${ }^{2}$ Assistant Professor of Radio-Diagnosis, Tanta University, Egypt.

${ }^{3}$ Consultant S.L.P. Certified and Instructor vitalstim therapy, USA.

${ }^{4}$ Medical Student, Faculty of medicine, Cairo University, Egypt.
}

\begin{abstract}
Aim: Of our study is to evaluate the efficacy of transcutaneous electrical stimulation (Vitalstim therapy) to treat patients with dysphagia and to determine the impact of the modified barium swallow study (MBSS) on patient management.

Material and methods: From June 2014 to April 2016, seventy three patients with dysphagia were included. All the patients underwent pre vitalstim-therapy evaluation by speech-language pathologists, including clinical evaluation of swallowing and modified barium swallow study (MBSS). In MBSS we evaluate the following, delay in oropharyngeal swallowing phase, closure of epiglottis, laryngeal elevation, passage of contrast behind the epiglottis that denoting penetration, presence of aspiration and presence residual barium after swallowing. After VitalStim therapy, all patients underwent MBSS assessments and also underwent a follow up survey months (range, 1 to 6 months) after their therapy to assess whether the improvement was worthwhile and sustained.

Results: In our study our 73 patients showing radiological findings denoting neurological swallowing disorders, 65 patients (89\%) showing delay in oropharyngeal swallowing phase, weak laryngeal elevation found in 58 patients $(79 \%)$, 68 patients (93\%) showing aspiration and 70 cases $(96 \%)$ showing penetration and incomplete closure of the epiglottis. Presence of residual barium after swallowing within the vallecula was seen in 68 patients (93\%). 52 of the 73 patients (71\%) showing complete improvement at first follow up study after VitalStim therapy. 21 of the 73 patients (29\%) showing some improvement and need another therapy sessions and in the next follow up study 15 cases showing complete improvement. However, 7 cases of the 21 patients having severe dysphagia before therapy, only 2 of 7 showed any improvement, and these patients still required a feeding tube for adequate nutrition, however, the 5 of 7 showing complete improvement after another session of therapy.
\end{abstract}

Conclusion: We conclude that the modified barium swallow is valuable in the rehabilitation of patient with swallowing disorders helps speech pathologist to identify and modify swallowing abnormalities as well as management program. We also conclude that VitalStim therapy is effective and safe in the treatment of patients suffering with the swallowing difficulties.

Keywords: Deglutition, dysphagia, modified barium swallow, vitalstim therapy, electrical muscle stimulation

\section{Introduction}

Deglutition is a highly integrated process of neural signaling and coordinated muscular contraction that begins with bolus preparation in the oral cavity and ends with closure of the lower esophageal sphincter after bolus passage [1].

Dysphagia is defined as difficulty swallowing and affects nearly 15 million adults in the United [2]. It is not a medical disease, per se, but a symptom or side effect of various neurological, structural, or cognitive conditions or deficits. It may be the result of head and neck trauma, cerebrovascular accident, neuromuscular degenerative diseases, head and neck cancer, and encephalopathies. 
Children and infants can have swallowing disorders as a result of a birth defect, or of an acquired birth defect, or of an acquired or developmental neurologic disorder $[3,4]$.

Patients often complain of coughing, food sticking and throat clearing when eating, feeling of something remaining in their mouth or throat after swallowing, and other discomfort related to eating [5].

Dysphagia leaves patients at risk for aspiration, because the muscles and nerves surrounding the oropharynx are not able to aid in safely transporting food from the mouth into the stomach. Aspiration can then lead to aspiration pneumonia, which accounts for approximately $34 \%$ of stroke related deaths, as well as cause complications such as choking, bronchospasm, increased infection rate, dehydration and nutritional compromise [6].

About $20-30 \%$ of patients with dysphagia silently aspirate which is when no outward signs or symptoms of aspiration, such as coughing or throat clearing, are present when food/ liquids enter the airway. The incidence of silent aspiration is higher with patients who have dysphagia following a stroke and is reported at $28-39 \%$.

There are many techniques can be used to treat dysphagia, include compensatory strategies, diet modifications, thermaltactile stimulation, and oral motor exercises [2].

VitalStim Therapy is a unique dysphagia therapy using neuromuscular electrical stimulation (NMES) to stimulate the muscles that are active during the swallow.

It gives a hope for those who have been suffering with dysphagia with a chance to improve quality of life. It was approved by the US Food and Drug Administration in 2001 for the treatment of dysphagia through the application of neuromuscular electrical stimulation to cervical swallowing muscles [7].

VitalStim is the only therapy technique that stimulates the swallowing muscles using an external electrical stimulation. The use of electrical stimulation has been practiced in the field of physical therapy for many years but has just recently been introduced to the treatment of swallowing disorders [2].

VitalStim can improve dysphagia therapy then the patients should be able to swallow more effectively, therefore reducing the number of illnesses and deaths caused by dysphagia (1a).

Impact of VitalStim therapy includes the following; correcting dysphagia at early stages of the condition, preventing its progression to the point where the patient may require a feeding tube and enabling many patients on tubes to return to normal or partly-normal eating.

The modified barium swallow is a radiographic (videofluoroscopic) procedure,that designed to define the anatomy and physiology of the patient's oropharyngeal swallow phase and also examine the effectiveness of rehabilitation treatment designed to eliminate aspiration and excess oral or pharyngeal residue [7].

Unlike the routine barium swallow in which only the esophageal stage of swallowing is studied, using the modified barium swallow all four stages of swallowing are studied with particular emphasis on the oral and pharyngeal stages [9]. It is a fluoroscopic procedure designed to determine whether food or liquid is entering a person's lungs, also known as aspiration.

Combining the modified barium swallow study with a follow-up swallowing rehabilitation plan (VitalStim therapy) will decrease the time as well as the cost for rehabilitation of patients with dysphagia [7].

\section{Materials and methods}

Our study was carried out by including 73 subjects, presenting to Radiology department with difficulty in swallowing. All the patients underwent pre-VitalStem therapy and postVitalStem therapy MBSS. Follow up survey months (range, 1 to 6 months) after their therapy to assess the improvement of dysphagia and if it was worthwhile and sustained.

The approval for our study was taken from our hospital Human ethics committee.

\section{The modified barium swallow (MBSS)}

The modified barium swallow study (MBSS) or videofluoroscopic examination is a moving $x$-ray of the oral and pharyngeal aspects of swallow and can be extended to view the esophagus. In this test, the patient is given measured amounts of thin liquids ( $1 \mathrm{ml}, 3 \mathrm{ml}, 5 \mathrm{ml}, 10 \mathrm{ml}$, cup drinking), semisolid food (pudding), and solid food that requires chewing (usually a piece of cookie) to chew and swallow so that we can define abnormalities in the swallow. This test enables us to observe what parts of the swallow are normal and which muscles are functioning normally or abnormally. In infants we used milk only. The test takes approximately 15 minutes and involves very little $x$-ray exposure [10-13].

\section{Electrical muscle stimulator (VitalStim therapy)}

In VitalStim therapy electrodes are simultaneously activated over the submental and laryngeal regions, with the aim of stimulating a simultaneous contraction of the mylohyoid in the submental region (to elevate the hyoid bone) and the thyrohyoid in the neck (to elevate the larynx to the hyoid bone) (Ludlow et al., 2006) [14].

There is only one electrical stimulation device in the United States that is approved by the Food and Drug Administration (FDA, it is approved in June 2001 for the treatment of swallow disorders in children and adults (FDA, 2001) [15].

The course of treatment with electrical stimulation therapy (Vitalstim therapy) will given daily ( 12 sessions), followed by MBSS, if moderate or no improvement obtained, another course of treatment will given daily (12 sessions). Another MBSS will done after 2 nd course of treatment and if moderate or no improvement obtained, last course of treatment will given daily (12 sessions).

\section{Statistical analysis}

Data was evaluated by using statistical package for social sciences (SPSS) software version 10 for calculating percent- 
ages and frequencies.

\section{Results}

All our patients underwent pre-Vitalsim therapy and postVitalstim therapy modified barium swallow study (MBSS).

55 patients were females and 18 patients were males. Females to males' ratio was 3:1. The youngest patient in our study was 6 months old and the oldest patient was 90 years old. The mean age of the patients was $16 \pm 35$ years.

We had 6 patients below the age of 10, 3 of them showed improvement after first sessions; 2 after second session and only one patient needed third session to improve his swallowing function.

We found that 73 patients show radiological findings denoting neurological swallowing disorders, 65 patients showing delay in oropharyngeal swallowing phase, weak laryngeal elevation found in 58 patients, and 70 patients showing penetration (Figures 1A, 2A and $\mathbf{3 A}$ ) and incomplete closure of the epiglottis. Presence of residual barium after swallowing within the vallecula was seen in 68 patients (Figures 1B, 2B and $\mathbf{3 B}$ ). 68 cases showing aspiration (Figures 1 C, 2 C and $\mathbf{3 B}$ ).

First course of treatment with electrical stimulation therapy was given daily ( 12 sessions) until the criteria for improved swallowing were fulfilled. 52 of the 73 patients $(71 \%)$ showed marked improvement at the first course of treatment (Figure 1D). The duration of the therapy varied from 12 days in patients with moderate dysphagia and reaching 24 to 36 days in patients with severe dysphagia that need second or third course of treatment. The number of treatment sessions ranged from 12 to 36 sessions.
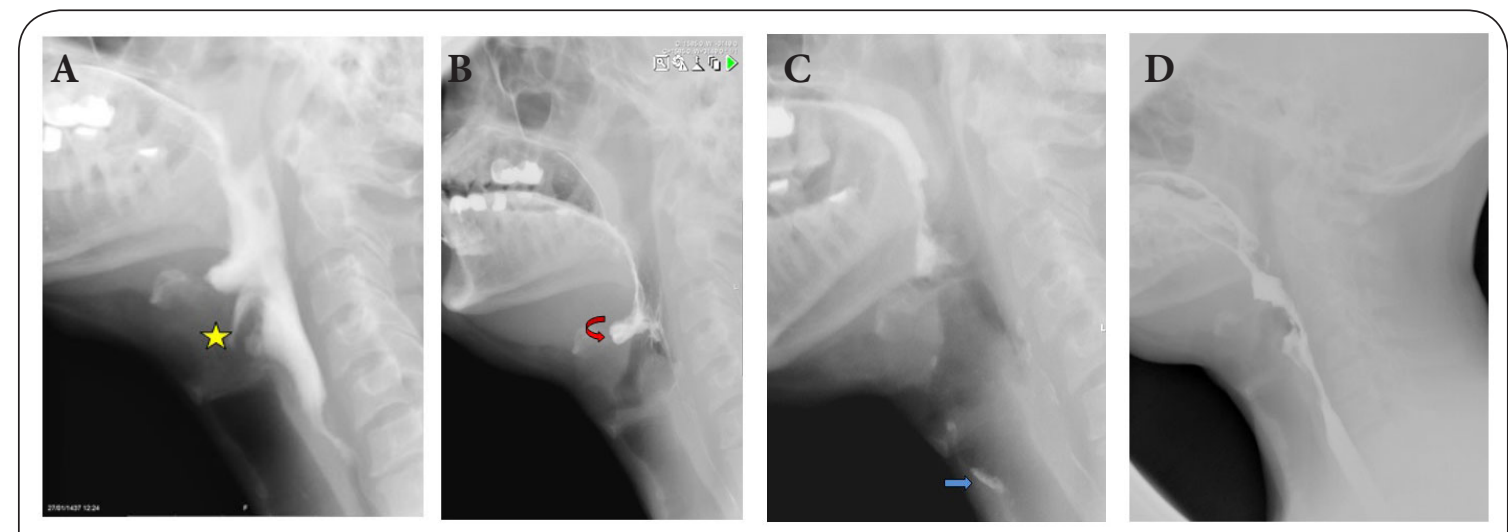

Figure 1.55 years old male patient.

MBSS revealed radiological features denoting neurological swallowing disorder including penetration (yellow star-A), with residual barium after swallowing (red curved arrow-B). and aspiration (blue straight arrow-C). (D). After Vitalstim therapy, follow up MBSS revealed marked improvement, include; Absence of penetration, absence of aspiration with improvement in the oropharyngeal swallowing phase and decrease in the amount of residual barium after swallowing.
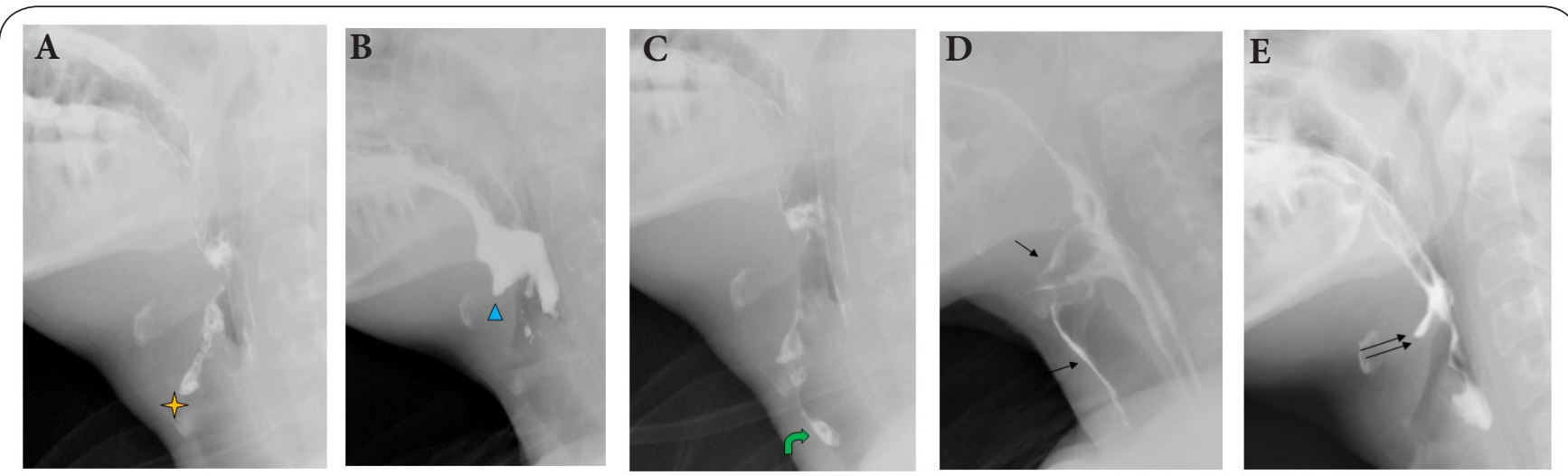

Figure 2. 54 years old male CVA patient.

MBSS revealed radiological features denoting neurological swallowing disorder including delay in the oropharyngeal swallowing phase, penetration (orange star-A), with residual barium after swallowing (blue triangle-B) and aspiration (green curved arrow-C). (D). After Vitalstim therapy, follow up MBSS revealed notable improvement in oropharyngeal phase \& improvement of basal tongue retraction. However, penetration, aspiration and residual barium within the vallecula after swallowing are still seen (black arrows). (E). In next follow up, MBSS revealed absence of aspiration with fluid and presence of penetration (double black arrows). 


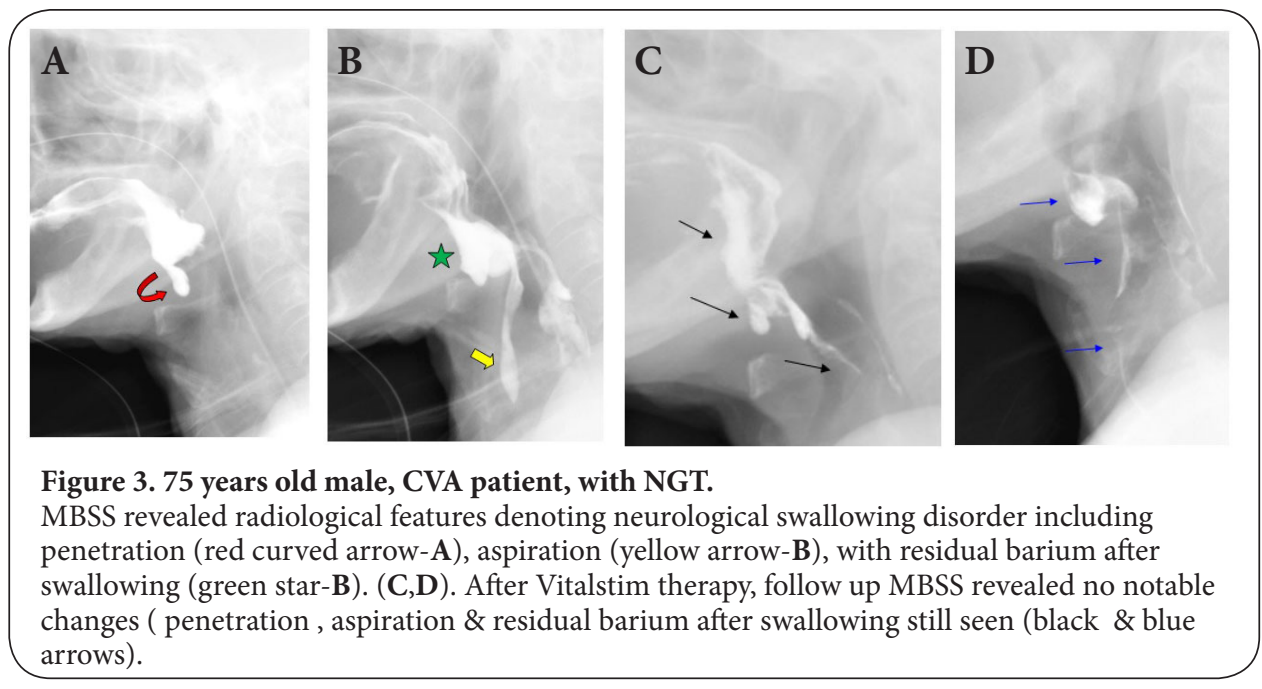

21 of the 73 patients (29\%) showing some improvement (Figure 2D) and need second course of treatment therapy (Figure 2E), and in the next follow up study 15 cases showing complete improvement. However, 7 cases of the 21 patients categorized as having severe dysphagia before therapy, only 2 of 7 showed any improvement (Figures $3 \mathrm{C}$ and $3 \mathrm{D}$ ) , and these patients still required a feeding tube for adequate nutrition, however, the 5 of 7 showing complete improvement after another session of therapy.

In our study, 5 patients had cardiac pacemakers, 10 of them showing mild skin irritation due to tape used to secure the stimulation electrodes to the anterior aspect of the neck.

Associated health problems includes diabetes in 20 patients (27\%) , hypertension 25 patients (34\%) , chronic obstructive pulmonary disease (COPD) or coronary heart disease in 19 patients (26\%) , sever metabolic disorders seen in 9 patients (12\%), Down syndrome in 2 patients (2\%) and epilepsy in 4 patients (5\%).

$31(42 \%)$ of our patients had nasogastric tubes, $28(38 \%)$ ofthem in cerebrovascular accident (stroke). 15 patients with unilateral hemispheric strokes, and 13 patients with bilateral hemispheric strokes. Pneumonia developed in 12 of $28 \mathrm{pa}-$ tients with stoke.

From our study we found that VitalStim Therapy is effective independently of the cause of a patient's dysphagiaas 52 of the 73 patients $(71 \%)$ showed marked improvement at the first course of treatment. Also it was effective in both sexes, 43 of 52 patients were females and 9 patients were males. The Vitalstim therapy was effective regardless of the time from dysphagia onset to rehabilitation therapy; the average time was $48 \pm 8$ days.

\section{Discussion}

Effective swallowing is very important for maintaining adequate nutrition, hydration, and quality of life and it is a very complex neuromuscular action requiring rapid and precise coordination of numerous cranial nerves and muscle pairs. Impairments of that action have the potential to confound and even precede medical complications, and reduce quality of life in patients.

Aspiration, is an inhalation of food/liquid or gastric contents passing through the vocal cords, can be a serious complication of dysphagia. It can cause coughing, choking, airway obstruction, and if the aspirated material reaches the lungs can result in aspiration pneumonia.

Modified Barium Swallow (MBS) is a fluoroscopic procedure designed to determine whether food or liquid is entering a person's lungs (aspiration) and it is a dynamic technique designed to evaluate swallowing function and dysfunction as it relates to the oral and pharyngeal phases of swallowing.

It permits the medical team to observe the coordination of anatomical structures in the mouth and throat, as they are actively functioning when chewing, drinking, and swallowing. It also identifies the reason for aspiration.

MBS is an analysis of swallowing through 3 phases: oral (mouth), pharyngeal (throat) and upper esophagus. It enables the speech pathologist and the physician to identify more clearly the reason or etiology of dysphagia, rule out aspiration and make decisions regarding management of the patient's swallowing disorder. The speech pathologist needs a specific etiology identified before swallowing treatment can be initiated. A treatment plan is developed for each patient specific to individual needs.

VitalStim therapy is non-invasive. The treatment uses controlled neuromuscular electrical stimulation to strengthen the muscles used in swallowing.It is used to repeatedly contract muscles by passing electrical currents through electrodes in contact with the body. The electrodes are attached to the stimulator device, which may be powered by battery or line voltage and controls the amount of energy flowing through the electrodes [13].

It is contraindicated in patients with significant reflux due to use of a feeding tube and in patients with dysphagia due to drug toxicity because of the risk of aspiration during trials of oral intake. 
As in our study, the study conducted by Freed et al., (2001) [16] and a case study by Sabet et al., (2005, Level 2) [17], found that the usage of electrical stimulation for dysphagia is a safe technology.

However, another study by Suiter (2006, Level 8) [18] showed that there was no significant gains in myoelectric activity of the sub mental muscles following neuromuscular Electrical Stimulation. Therefore, the benefit of Neuromuscular Electrical Stimulation to the sub mental muscles with the goal of improving the pharyngeal swallow is not supported.

On the other hand, Chetney \& Waro (2004) [19] evaluated the VitalStim device and found that it could be utilized by speech therapists in a home care setting.

Freed et al., (2001) [16] reported that the electrical stimulation using VitalStim was $97 \%$ successful in improving or restoring a patient's ability to appropriately and adequately swallow.

In another study by Leelamanit et al., (2002) [20], the authors concluded that, stimulating synchronous contraction of the thyrohyoid muscle by the electrical stimulator during swallowing improves dysphagia (resulting from reduced laryngeal elevation).

We found that VitalStim Therapy was effective across the full spectrum of age groups and in both sexes, also it was effective independently of the cause, associated with health problems. Also, we found that it is effective independently of the time between the onset of dysphagia and the treatment.

The results of this study showed significant improvement in overall swallow function after treatment with VitalStim therapy.

From the follow up survey we confirm the consistent improvement in swallow function after therapy.

\section{Conclusion}

The modified barium swallow is a valuable in the rehabilitation of patient with swallowing disorders allowing speech pathologist to identify and modify swallowing abnormalities.

Neuromuscular Electrical stimulation for dysphagia is relatively a new technology and it is non-invasive and safe for the treatment of dysphagia.

Further randomized controlled trials are needed to demonstrate the clinical benefits of electrical stimulation for dysphagia and to help clinicians to learn the value and limitations of vital -stim therapy.

\section{Competing interests}

The authors declare that they have no competing interests.

Authors' contributions

\begin{tabular}{|l|c|c|c|c|}
\hline Authors' contributions & HSD & HAK & MAJ & MYH \\
\hline Research concept and design & $\checkmark$ & -- & -- & -- \\
\hline Collection and/or assembly of data & $\checkmark$ & $\checkmark$ & $\checkmark$ & -- \\
\hline Data analysis and interpretation & $\checkmark$ & $\checkmark$ & -- & -- \\
\hline Writing the article & $\checkmark$ & -- & -- & $\checkmark$ \\
\hline Critical revision of the article & $\checkmark$ & $\checkmark$ & -- & $\checkmark$ \\
\hline Final approval of article & $\checkmark$ & $\checkmark$ & $\checkmark$ & $\checkmark$ \\
\hline Statistical analysis & $\checkmark$ & -- & -- & -- \\
\hline
\end{tabular}

\section{Acknowledgement}

The authors would like to thank their colleagues for the support and patients for their trust.

Publication history

EIC: Domenico Rubello, Santa Maria della Misericordia Hospital, Italy. Received: 21-Oct-2016 Final Revised: 09-Dec-2016

Accepted: 05-Jan-2017 Published: 20-Jan-2017

\section{References}

1. Gullung JL, Hill EG, Castell DO and Martin-Harris B. Oropharyngeal and esophageal swallowing impairments: their association and the predictive value of the modified barium swallow impairment profile and combined multichannel intraluminal impedance-esophageal manometry. Ann Otol Rhinol Laryngol. 2012; 121:738-45. | PubMed

2. Nass LB. VitalStim: Using Electrical Stimulation Therapy to Improve Dysphagia Symptoms in Adults. 2011. I Website

3. Arvedson J and Lefton-Greif M. Pediatric Videofluoroscopic swallow studies. San Antonio (TX): The Psychological Corporation. 1998.

4. Logemann JA. Evaluation and treatment of swallowing disorders. 2nd ed. Austin (TX): Pro Ed. 1998.

5. Logemann JA. Dysphagia (Difficulty swallowing or difficulty moving food from mouth to stomach). In: JH Stone, M Blouin, editors. International Encyclopedia of Rehabilitation. 2010.

6. Lim KB, Lee HJ, Lim SS and Choi YI. Neuromuscular electrical and thermal-tactile stimulation for dysphagia caused by stroke: a randomized controlled trial. J Rehabil Med. 2009; 41:174-8. | Article | PubMed

7. Keohane J, Jr., Lampe HB and Poluha P. Use of the modified barium swallow in the rehabilitation of the swallowing mechanism. J Otolaryngol. 1988; 17:368-71. | Article | PubMed

8. Logemann JA. Role of the modified barium swallow in management of patients with dysphagia. Otolaryngol Head Neck Surg. 1997; 116:335-8. | Article I PubMed

9. Shaw GY, Sechtem PR, Searl J, Keller K, Rawi TA and Dowdy E. Transcutaneous neuromuscular electrical stimulation (VitalStim) curative therapy for severe dysphagia: myth or reality? Ann Otol Rhinol Laryngol. 2007; 116:36-44. | PubMed

10. Beck TJ and Gayler BW. Image quality and radiation levels in videofluoroscopy for swallowing studies: a review. Dysphagia. 1990; 5:118-28. | Article | PubMed

11. Chan CB, Chan LK and Lam HS. Scattered radiation level during videofluoroscopy for swallowing study. Clin Radiol. 2002; 57:614-6. | Article | PubMed

12. Logemann JA. A manual for videofluoroscopic evaluation of swallowing. 2nd ed. Austin (TX): Pro Ed. 1993.

13. Wright RE, Boyd CS and Workman A. Radiation doses to patients during pharyngeal videofluoroscopy. Dysphagia. 1998; 13:113-5. | Article | PubMed

14. Ludlow CL, Humbert I, Saxon K, Poletto C, Sonies B and Crujido L. Effects of surface electrical stimulation both at rest and during swallowing in chronic pharyngeal Dysphagia. Dysphagia. 2007; 22:1-10. | Article | PubMed Abstract | PubMed FullText

15. FDA.

16. Freed ML, Freed L, Chatburn RL and Christian M. Electrical stimulation for swallowing disorders caused by stroke. Respir Care. 2001; 46:466-74. | Pdf | PubMed

17. Sabet, Farzad MD, Williams, Jeffrey MD and Tolat Raj. Poster Board 110: Electrical Stimulation For Dysphagia: A Case Study And Review. Lippincott Williams \& Wilkins, 2005; 84:231. | Article

18. Suiter DM, Leder SB and Ruark JL. Effects of neuromuscular electrical stimulation on submental muscle activity. Dysphagia. 2006; 21:56-60. I Article | PubMed 
Darwish et al. Medical Imaging and Radiology 2017,

http://www.hoajonline.com/journals/pdf/2054-1945-5-1.pdf

19. Chetney R and Waro K. A new home health approach to swallowing disorders. Home Healthc Nurse. 2004; 22:703-7. | Article | PubMed

20. Leelamanit V, Limsakul C and Geater A. Synchronized electrical stimulation in treating pharyngeal dysphagia. Laryngoscope. 2002; 112:2204-10. | Article | PubMed

\section{Citation:}

Darwish HS, Kamel HA, Jeady MAL and Habash

MY. Role of modified Barium Swallow Pre and Post

VitalStim therapy In the rehabilitation of the swallowing mechanism. Med Imaging Radiol. 2017; 5:1.

http://dx.doi.org/10.7243/2054-1945-5-1 